\section{Regards sur l'économie allemande}

Bulletin économique du CIRAC

$100 \mid 2011$

Varia

\title{
20 ans après : la France et l'Allemagne dans la nouvelle Europe
}

Joachim Schild

\section{(2) OpenEdition}

\section{Journals}

Édition électronique

URL : http://journals.openedition.org/rea/4219

DOI : $10.4000 /$ rea.4219

ISBN : 978-2-8218-0894-2

ISSN : 1965-0787

\section{Éditeur}

CIRAC

\section{Édition imprimée}

Date de publication : 17 mars 2011

Pagination : 11-25

ISSN : 1156-8992

\section{Référence électronique}

Joachim Schild, « 20 ans après : la France et l'Allemagne dans la nouvelle Europe », Regards sur l'économie allemande [En ligne], 100 | mars 2011, mis en ligne le 01 mars 2012, consulté le 01 mai 2019. URL : http://journals.openedition.org/rea/4219; DOI : 10.4000/rea.4219 


\section{0 ans après : la France et 1'Allemagne dans la nouvelle Europe}

\section{Joachim Schild}

Le lancement de la revue Regards sur l'économie allemande en 1991 se situe dans le contexte historique de la chute du Mur de Berlin en novembre 1989, de la réunification allemande en octobre 1990 et de l'éclatement de l'Union soviétique fin 1991. Cette succession de séismes politiques au plan allemand, européen et international ne pouvait pas ne pas provoquer une multitude d'interrogations : sur la place future de cette Allemagne unifiée en Europe, sur ses relations avec son partenaire privilégié en Europe qu'est la France, ainsi que sur le rôle du bilatéralisme franco-allemand au sein de la construction européenne.

Avec le recul de deux décennies, nous pouvons jeter un regard sur la pertinence de certaines de ces questions de l'époque quant à la place des relations francoallemandes dans la nouvelle Europe.

A l'époque, un bon nombre d'observateurs et d'acteurs politiques français partaient de l'idée que l'Allemagne unifiée serait capable de digérer assez rapidement les coûts financiers de l'intégration économique de l'ex-RDA. L'unification augmenterait donc non seulement les ressources démographiques de son pouvoir, mais aussi le poids économique qu'elle pourrait mettre sur la balance en Europe. Appuyée sur son poids économique et mettant à profit ses liens historiques et culturels avec les pays de l'Europe centrale et orientale, la RFA serait tentée de renouer avec ses rêves d'une "Mitteleuropa" sous influence allemande, datant d'une autre époque. Dans tous les cas, elle prendrait une place incontournable dans cette Europe reconfigurée, la place d'une "Zentralmacht " (Schwarz, 1994) - d'une puissance centrale aussi bien au sens géographique que politique du terme. Cette Allemagne n'abandonnerait-elle pas petit à petit son profil bas en matière de politique étrangère, sa culture de retenue et d'autolimitation de ses ambitions? N'adopterait-elle pas un style de politique étrangère plus décomplexé, plus 'musclé', bref plus 'normalisé' à l'instar d'une puissance moyenne telle que la France et le Royaume-Uni? Et, finalement, cette Allemagne, n'aurait-elle pas plus d'options diplomatiques en Europe que de miser avant tout sur une "relation privilégiée " avec la France dans le cadre de l'Union européenne, s'appuyant sur des coalitions mouvantes au sein d'une Union européenne élargie et en établissant des liens stratégiques avec la Russie?

La France, quant à elle, ne se trouverait-elle pas reléguée au second rang en Europe, géographiquement - et aussi politiquement - à la périphérie du continent européen réunifié ? Ses atouts de puissance sur la scène mondiale - son statut de vainqueur de la Seconde Guerre mondiale et sa bombe atomique - n'étaientils pas dévalorisés?

Les années cinquante et surtout les années soixante du vingtième siècle étaient marquées par une place centrale et prépondérante de la France au sein de la politique ouest-européenne et dans le cadre des relations franco-allemandes. Puis, les deux décennies depuis la fin des années soixante jusqu'à la fin de la guerre froide étaient caractérisées par une sorte d'équilibre des déséquilibres entre la puissance politico-militaire de la France et la puissance économico-monétaire de la RFA. Cette nouvelle époque qui s'est ouverte en 1990, verrait-elle un glisse-

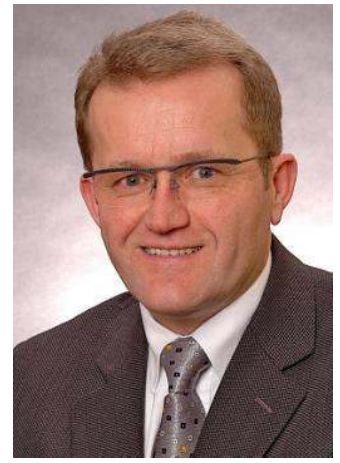

Joachim Schild (1962), Prof. de science politique de Trèves

depuis la fin 2003, avait été auparavant chargé de recherche à 1'Institut Franco-Allemand de Ludwigsburg (DFI) de 1990 à 2002. 
Une Allemagne européenne vs. une Europe-puissance

Un intérêt commun : préserver l'UEM

Rapprochement dans le discours et l'action politiques ment vers une hégémonie allemande au sein du "couple » franco-allemand, et au-delà, dans cette Europe nouvelle ? Et même au cas où la qualité des relations bilatérales pourrait être maintenue et développée, la force commune d'entraînement du tandem franco-allemand au sein d'une Union européenne qui s'élargit sans cesse, ne se trouverait-elle pas forcément diminuée?

Avec le recul qui est le nôtre aujourd'hui, il nous est possible de donner des éléments de réponse à certaines de ces questions. Dans cet article, nous nous proposons d'évaluer la qualité des relations franco-allemandes pendant ces deux décennies, mais aussi la fonction qui fut et qui est la leur au sein de l'Union européenne. Pour ce faire, nous analysons les défis au plan européen et international et au plan bilatéral, et la manière dont les deux pays ont su y faire face. Mais d'abord, il peut paraître utile de rappeler quelques grandes lignes de continuité de la politique européenne et bilatérale des deux voisins.

\section{Continuités malgré tout}

Ce qui frappe tout d'abord en portant le regard sur l'ensemble de cette période 1990-2010, c'est le contraste entre l'ampleur de la rupture du système international que représente la fin du conflit Est-Ouest et la continuité fondamentale des politiques européennes française et allemande.

Les motifs qui sous-tendaient la politique européenne de la France et de la RFA depuis les débuts de la construction européenne n'ont rien perdu de leur valeur et guident toujours l'action des responsables des deux côtés du Rhin. Au motif français de contrôler et d'ancrer la puissance allemande dans un cadre européen solide correspond toujours une volonté d'auto-ancrage de l'Allemagne. Celle-ci a besoin de s'arrimer solidement aux structures européennes pour éviter des réactions des voisins européens visant à équilibrer sa puissance économico-politique au milieu du continent. Une Allemagne européenne et non pas une Europe allemande, tel était le 'mantra' maintes fois répété par le gouvernement Kohl-Genscher. II sert toujours de fil conducteur à la diplomatie allemande. A l'idée française que l'Union européenne peut servir de multiplicateur de puissance pour sa diplomatie correspond toujours l'idée allemande qu'une diplomatie active passe nécessairement par des cadres multilatéraux, dont celui de l'Union prend une place centrale, si elle veut peser sur les affaires du monde et si elle veut éviter de susciter un malaise du côté de ses partenaires européens.

Les intérêts économiques fondamentaux des deux pays n'ont pas changé non plus : leur prospérité dépend essentiellement du bon fonctionnement du marché unique européen vers lequel la France exporte, selon Eurostat, $62 \%$, et la RFA $63 \%$ de toutes ses exportations de marchandises, tandis que $69 \%$ des importations françaises de marchandises et $65 \%$ des importations allemandes proviennent des partenaires de l'Union européenne en 2009. Les deux pays clés de la zone euro partagent également le même intérêt fondamental à préserver le bon fonctionnement de celle-ci. Paris et Bonn furent les deux principaux maîtres d'œuvre de son lancement depuis 1988 et se trouvaient au cœur des négociations de Maastricht en 1991. Aujourd'hui, Paris et Berlin se trouvent toujours au cœur des décisions politiques au niveau européen pour contenir la crise de la dette souveraine et pour redéfinir des règles du fonctionnement de la zone euro. Ceci reflète leur intérêt commun - et fondamental - de préserver la zone euro, une communauté de destin dont dépend essentiellement leur prospérité économique.

Les modes d'action de la politique européenne française et allemande et les discours européens des deux pays ont certes connu des évolutions au cours des vingt dernières années. Mettre l'accent sur les « intérêts nationaux » qui peuvent diverger de l'intérêt général européen n'est plus un tabou en RFA (et n'était jamais un tabou en France). A plusieurs reprises, au cours de ces vingt dernières années, la France et l'Allemagne n'ont pas hésité à s'affronter directement au 
niveau européen et à étaler leurs désaccords au grand public, aussi bien au cours de la négociation du Traité de Nice qu'au début de la crise financière en 2008. Leur volonté commune d'approfondir l'Union européenne n'est plus la même qu'au début des années 1990. Des transferts de compétences, voire de souveraineté au niveau européen se heurtent aujourd'hui à des contraintes en matière de politique intérieure: des opinions publiques plus sceptiques qu'au début des années 1990 et, du côté de la RFA, des Länder nettement plus enclins à défendre leurs intérêts en politique européenne vis-à-vis du gouvernement fédéral ou encore une cour constitutionnelle à Karlsruhe qui a tracé des bornes juridiques à la politique européenne de la RFA à travers ses arrêts sur le Traité de Maastricht en 1993 et surtout sur le Traité de Lisbonne en 2009. Des grands projets d'intégration poussés par la France et la RFA sont difficiles à trouver ces dernières années. Et les grands rêves fédéraux en Allemagne semblent bel et bien derrière nous. Le mot d'ordre d'aujourd'hui, c'est la consolidation des acquis en matière de construction européenne, comme le révèle par exemple un article publié le 9 mai dans le quotidien Frankfurter Allgemeine Zeitung par Volker Kauder, président du groupe parlementaire CDU/CSU au Bundestag.

II n'empêche, leurs élites politiques et les partis de gouvernement (CDU/CSU, FDP, SPD, Verts) partagent une conviction toujours bien ancrée : la France et la RFA n'ont une solution de rechange crédible ni à la poursuite de leur politique en matière de construction européenne, ni à une coopération bilatérale étroite, qui est toujours largement vue comme étant le meilleur garant de la prise en compte de leurs intérêts au niveau européen et international.

Les divergences de vue et d'approche parfois profondes face aux défis extérieurs et intérieurs que nous nous proposons d'aborder ci-après sont donc à interpréter sur fond de cette continuité des intérêts et orientations fondamentaux des deux pays.

Les irritations bilatérales au moment de la chute du Mur et au cours de la marche vers l'unification allemande une fois passées (voir par exemple Bozo, 2005), les politiques européennes de la France et de l'Allemagne réunifiée ont dû faire face à un certain nombre de défis internes et externes. Au plan interne, l'approfondissement de la Communauté européenne était à l'ordre du jour pratiquement sans arrêt, depuis les négociations sur le Traité de Maastricht (1990/91) jusqu'en 2009. Au plan des défis externes, ce sont surtout les crises yougoslaves dans les années 1990 et la guerre irakienne en 2003, ainsi que le processus de stabilisation, de transformation et de démocratisation des pays postcommunistes et leurs demandes d'adhésion à l'Union européenne qui ont dominé les agendas politiques. Dans plusieurs de ces dossiers, les approches de la France et de l'Allemagne étaient différentes, voire antagonistes au début pour se rapprocher au fur et à mesure de l'évolution de l'agenda européen et international.

\section{Le défi interne : approfondissement de la construction européenne}

L'Acte unique européen, en vigueur depuis 1987, marque le début d'un long cycle de réformes des traités et des institutions de la Communauté européenne, devenue Union européenne en 1993, et qui fut seulement achevé (provisoirement) avec l'entré en vigueur du Traité de Lisbonne (2009). Le rôle qui fut celui de la France et de l'Allemagne dans ce processus de réformes était souvent crucial, aussi bien pour les avancées sur la voie d'un approfondissement de la construction européenne que pour les échecs et demi-échecs en cours de route.

Trois sources principales ont alimenté ce long processus de réformes : l'hégémonie monétaire de la RFA au sein du Système Monétaire Européen (SME), l'unification allemande et la perspective de l'élargissement de l'Union. Mettre fin à l'hégémonie de la RFA en matière de politique monétaire européenne et à la place dominante qui fut celle du deutsche mark au sein du SME est devenu une priorité de la politique européenne de la France depuis 1988. La RFA a, elle
Continuité des intérêts...

... malgré des divergences de vue parfois profondes 
Intégration monétaire : une suite de compromis

«Union politique »: deux approches

Amsterdam et Nice. La question de la représentativité aussi, donné dès 1988 son accord de principe à la perspective d'une intégration monétaire approfondie. Ensuite, la chute du Mur et l'unification allemande ont donné une impulsion importante aux réformes du droit primaire de l'Union, débouchant sur le Traité de Maastricht dont l'élément principal fut la marche vers I'Union Economique et Monétaire (UEM). La troisième force de propulsion de ces réformes des traités fut la perception d'une nécessaire préparation institutionnelle de l'Union à son l'élargissement à l'Est.

Le rôle commun de la France et de l'Allemagne, fermement soutenu par la Commission européenne sous l'autorité de Jacques Delors, fut décisif tout au long du processus d'intégration monétaire (voir l'ouvrage de référence de Dyson/Featherstone, 1999 ; voir aussi le récit passionnant de Marsh, 2009). Ceci est aussi bien vrai pour l'inscription de cette dernière à l'agenda communautaire que pour la recherche des compromis essentiels. Quant au calendrier serré et contraignant pour ce saut historique vers l'UEM, c'est la position française qui a prévalu. Mais pour ce qui est du modèle institutionnel calqué sur celui de la Bundesbank allemande et garantissant l'indépendance de la Banque Centrale Européenne (BCE) vis-à-vis des institutions politiques, c'est bien la RFA qui a eu gain de cause. La même chose est vraie pour les objectifs assignés par le traité à la $\mathrm{BCE}$, notamment la priorité sans ambiguïté accordée à la lutte contre l'inflation, fût-ce au détriment de la croissance, et pour l'inscription d'une clause de «no bail out » dans le traité pour éviter que des pays 'vertueux' payent le prix d'une politique budgétaire irresponsable menée dans tel ou tel pays membre de la zone euro.

La France et l'Allemagne sont aussi les principaux responsables du caractère inégal de la poursuite de la construction européenne au début des années 1990. Le gouvernement Kohl-Genscher a plaidé en vain pour une « Union politique » en complément nécessaire de I'UEM. Ce cryptogramme d'« Union politique » renvoyait, au sens allemand, aussi bien à un caractère nettement plus coordonné, voire intégré, de la politique étrangère des pays membres, qu'à une fédéralisation du système politique européen, notamment une promotion du rôle du Parlement européen. Le président Mitterrand ne voulait ni de l'un ni de l'autre. La France fut à l'origine de l'idée de séparer très clairement la politique étrangère et de sécurité commune (PESC) des politiques communautaires, en inventant la structure en trois piliers de l'Union européenne. Et même si elle a dû faire des concessions sur le rôle du Parlement européen avec l'introduction de la procédure de codécision entre celui-ci et le Conseil en matière de législation, les avancées vers une Union politique restaient donc bien en deçà des attentes allemandes (cf. Mazzucelli, 1997).

Dans les étapes ultérieures des réformes des traités, les questions institutionnelles se trouvaient toujours au cœur du débat. Celui-ci fut d'autant plus difficile que les enjeux de pouvoirs qui sous-tendent toute réforme institutionnelle n'étaient plus, comme dans le passé, liés à un projet d'intégration d'ampleur comme le furent le marché unique ou l'Union monétaire. Au cours des négociations en vue des traités d'Amsterdam et de Nice, la France et l'Allemagne n'ont pas réussi à jouer un rôle de force de proposition et de facilitateur de compromis. A part leur engagement commun pour l'inscription de formes de coopérations renforcées au sein de groupes restreints de pays membres dans les deux traités, leurs priorités divergentes ont contribué au caractère très limité des deux réformes. La France insistait, en vain à Amsterdam, sur une réforme institutionnelle qui resserrerait la Commission, élargirait le nombre de politiques soumises au vote à la majorité qualifiée au Conseil et augmenterait le poids des grands pays au sein de celui-ci. Tout en partageant au fond ces objectifs, la RFA s'est rangée du côté des petits et moyens Etats membres qui ne voulaient pas céder du terrain en ce qui concerne leur représentation au sein des institutions communautaires, notamment au Conseil. La réforme institutionnelle et l'approfondissement de l'Union étaient à l'époque éclipsés par la priorité donnée par la RFA à l'élargissement de l'Union vers l'Est. 
Le débat institutionnel, et avec lui le clivage entre petits et grands pays de l'Union, est réapparu plusieurs fois par la suite, notamment à l'occasion des négociations du Traité de Nice, mais aussi lors des débats sur le Traité constitutionnel et le Traité de Lisbonne. Contrairement aux négociations d'Amsterdam, la RFA s'est associée par la suite à la France et au Royaume-Uni pour défendre les intérêts institutionnels et le pouvoir des grands pays dans une Union qui était sur le point de faire entrer neuf petits pays et un seul d'une taille plus importante (la Pologne).

Mais cette question de la représentation du poids démographique au sein des instances communautaires ne pouvait pas ne pas soulever la question de la parité franco-allemande au Conseil, étant donné que la RFA comptait à l'époque 20 millions d'habitants de plus que la France. L'obstination du président Jacques Chirac à défendre cet acquis historique de la parité franco-allemande au Conseil et la position ferme du chancelier Schröder sur cette question ont provoqué des conflits bilatéraux d'une profondeur qu'on n'a plus vue depuis les années soixante et la politique de la chaise vide du Général de Gaulle en $1965 / 66$. Pour la première fois depuis le début de ce cycle de réformes, la France et la RFA était incapables de soumettre la moindre proposition commune, et leur discorde bilatérale constituait une des pierres d'achoppement centrales sur le chemin de la recherche d'un compromis à Nice. Néanmoins, la France n'a pas pu empêcher un décrochage institutionnel par rapport à la RFA dont le poids démographique fut pris en compte dans la formule complexe pour réunir une majorité qualifiée au Conseil (voir Schild, 2001).

Les deux gouvernements ont cherché à trouver des moyens pour sortir par le haut de cette situation en institutionnalisant en 2001 un rythme accéléré de consultations informelles bilatérales au plus haut niveau - Président de la République, le Chancelier et les ministres des Affaires étrangères - : le processus dit de Blaesheim.

A partir du mois d'octobre 2002, après les élections en France et en Allemagne, le bilatéralisme franco-allemand en matière européenne a pu être rénové d'une manière spectaculaire à l'occasion de la célébration $\mathrm{du} 40^{\mathrm{e}}$ anniversaire du Traité de l'Elysée (22 janvier 2003) et au cours des travaux de la Convention européenne chargée d'élaborer un Traité constitutionnel. La France et l'Allemagne ont en effet retrouvé leur rôle de force de proposition et de compromis au cours de ce travail de refonte des traités fondateurs. C'est surtout sur le volet institutionnel que leurs contributions soumises au débat de la Convention ont eu le plus grand impact (voir Schild, 2004). Leur contribution commune sur l'architecture institutionnelle de l'Union, publiée en janvier 2003, contenait deux éléments clés, une structure bicéphale au sommet de l'Union - le président de la Commission et un personnage nouveau, le président à temps plein du Conseil européen - et l'architecture institutionnelle pour l'action extérieure de I'Union avec un ministre européen des Affaires étrangères. Le choix francoallemand d'une structure bicéphale au sommet a pu être qualifié de "compromis hégémonique » (Magnette/Nicolaïdis, 2003) et a de nouveau actualisé et approfondi le clivage entre grands et petits pays au sein de l'Union.

Une attitude de fermeté a pu être observée de la part de Paris et de Berlin à propos du dossier qui a fait échouer la première tentative d'arriver à un compromis parmi les vingt-cinq Etats membres en 2003, à savoir le système de votation au sein du Conseil. Les deux pays refusaient clairement la tentative des gouvernements espagnol et polonais de garder le système complexe du Traité de Nice et mettaient tout leur poids derrière le système d'une double majorité des Etats membres et de la population qu'ils représentent pour réunir une majorité qualifiée. Sur cette question, Paris et Berlin ont finalement eu gain de cause.

Après l'échec définitif du texte constitutionnel suite à l'issue des référendums en France et aux Pays-Bas en mai et juin 2005, la France et la RFA ont cherché des voies pour préserver l'essentiel de cette réforme, surtout après la clarifica-
Nice. Quelle parité

France/Allemagne?

Elaboration conjointe du projet de Traité constitutionnel 
Equilibre subtil entre éléments supranationaux et intergouvernementaux tion politique de l'élection présidentielle française en 2007. Ils ont adopté une posture 'défensive', afin d'éviter que d'autres gouvernements ne procèdent à un vaste 'détricotage' du texte constitutionnel, tout en cherchant des modifications qui permettraient un accord à 27 (voir REA 82/07).

Durant ce processus de réforme des institutions et des politiques de l'Union, les positions françaises et allemandes se sont rapprochées. Du côté allemand, le zèle fédéraliste est devenu certainement plus tempéré que par le passé. Les responsables allemands ne se sont pas battus pour une Commission au centre du dispositif européen, ils ont facilement accepté le caractère intergouvernemental de la PESC et la montée en puissance du Conseil européen. Et l'Allemagne a de plus en plus mis l'accent, depuis le début des années 1990, sur le respect du principe de subsidiarité et sur une délimitation très claire des compétences entre le niveau national et européen. Différents gouvernements allemands n'ont pas hésité depuis les négociations sur le Traité d'Amsterdam à défendre des morceaux de souveraineté nationale, surtout en matière d'immigration légale. La France, de son côté, a fait montre d'un nouveau pragmatisme en matière institutionnelle qui sert ses intérêts de fond. Depuis la rupture du président Mitterrand avec le dogme gaullien du vote à l'unanimité sur des dossiers importants, Paris a souscrit au passage au vote à la majorité qualifiée au Conseil dans un nombre croissant de domaines, de la politique industrielle et sociale jusqu'à la coopération en matière de politique intérieure et de justice. Développer des politiques européennes efficaces et faire prévaloir ses propres intérêts peut se révéler plus facile dans certains domaines au niveau européen si la règle décisionnelle est celle de la majorité qualifiée, selon l'analyse faite à Paris.

L'équilibre subtil entre des éléments intergouvernementaux et supranationaux dans le dispositif institutionnel de l'Union est non seulement le fruit historique d'une synthèse - ou parfois d'une simple juxtaposition - des idées constitutionnelles allemandes et françaises pour l'Europe (voir Scholl, 2006 ; Bitsch, 2001), il est aussi de plus en plus accepté des deux côtés du Rhin comme étant le reflet de la double nature de l'Union européenne en tant qu'Union des Etats et Union des citoyens.

\section{Les défis extérieurs : élargissement et gestion de crises}

L'agenda politique européen de ces vingt dernières années était dominé pendant de longues périodes par les dossiers des différentes réformes des traités qui ont capté l'attention des décideurs politiques. Mais les défis externes ne manquaient pas non plus, c'est le moins qu'on puisse dire dans ce monde en pleine mutation de l'après guerre froide.

La guerre en ex-Yougoslavie

Le premier grand test, “l'heure de l'Europe » selon la formule maladroite du ministre luxembourgeois de l'époque, Jacques Poos, fut celui de la guerre en exYougoslavie. L'échec de la politique européenne vis-à-vis de ce foyer de crise était presque total, surtout pendant les premières années jusqu'aux accords de Dayton en 1995, et les divergences profondes entre la France et la RFA y étaient pour beaucoup (voir Stark et al., 1995 ; Maul/Stahl, 2002).

Tandis que le président Mitterrand voyait dans ce conflit une « guerre de tribus» face à laquelle l'Union européenne devrait assumer le rôle d'un médiateur neutre, le gouvernement Kohl-Genscher, de son côté, mettait l'accent sur la guerre d'agression menée par la Serbie contre les Républiques sécessionnistes. Bonn œuvrait en faveur d'une internationalisation du conflit par une reconnaissance internationale de la Slovénie et de la Croatie pour endiguer les hostilités sans pour autant pouvoir ou vouloir intervenir elle-même militairement. Au cours de la guerre en Bosnie, les deux gouvernements ont cherché, non sans mal, à rapprocher leurs positions à travers des déclarations communes et des initiatives des ministres des Affaires étrangères de l'époque, Klaus Kinkel et Alain Juppé. Malgré des différences importantes, réapparues pendant le conflit au Kosovo en 1998/99, les deux gouvernements s'efforçaient de limiter l'effet de leurs diver- 
gences bilatérales pour qu'elles ne rejaillissent pas sur d'autres domaines de leurs relations bilatérales au sein de l'Union européenne. Ce réflexe de 'limiter la casse' souligne la permanence du caractère privilégié de cette relation bilatérale et la perception de sa valeur propre des deux côtés.

Le défi majeur en termes de politique extérieure de l'Union européenne durant toute cette période était sûrement celui de la stabilisation, de la transformation et finalement de l'intégration des pays postcommunistes de l'Europe centrale et orientale dans le cadre des structures européennes. Or, par rapport à cette question fondamentale du réaménagement de l'ordre européen, les approches de la France et de la RFA furent fort différentes au début pour se rapprocher par la suite (voir Deubner, 1999).

Dès ses vœux pour le nouvel an de 1990, le président Mitterrand avait lancé l'idée d'une confédération européenne. Celle-ci devrait permettre un rapprochement lent des pays de l'Europe centrale et orientale (PECO) à la Communauté européenne. L'approfondissement de I'Union avait clairement sa priorité avant tout processus d'élargissement. Cette position fut partagée par le gouvernement de Helmut Kohl jusqu'à la signature du Traité de Maastricht. Par la suite, Bonn se fit de plus en plus l'avocat fervent d'un élargissement rapide de I'Union européenne, objectif stratégique de première importance de sa politique étrangère. La RFA voulait mettre fin à sa situation à la périphérie de I'Union européenne, directement confrontée aux risques d'instabilité politiques, économiques et sociaux dans son voisinage immédiat, et à tous les problèmes qui peuvent en découler en termes de sécurité et de migration. La RFA n'avait nullement l'intention de 'monopoliser' les relations vers les pays postcommunistes de l'Est afin d'établir une zone d'influence exclusive. Tout au contraire, elle avait le plus grand intérêt à insérer sa politique envers l'Est dans le cadre multilatéral de la Communauté et à s'appuyer sur les instruments multiples de celle-ci pour favoriser une transition réussie des PECO vers la démocratie, l'Etat de droit et l'économie de marché.

Après l'échec définitif des projets français d'une confédération en 1993, faute d'intérêt des pays de l'Europe centrale et orientale, la France a dû s'ouvrir à la perspective d'un élargissement de l'Union. Un changement de l'attitude française s'est manifesté après l'arrivée au pouvoir du président Jacques Chirac. Celui-ci s'est livré à une course de vitesse avec le gouvernement allemand en termes de promesses (non tenues) faites aux pays candidats quant à leur date d'entrée. Néanmoins, la France se rangeait, jusqu'au moment du grand élargissement en mai 2004, dans le camp des sceptiques. A part les craintes que la RFA soit le grand profiteur politique et économique de cet élargissement vers l'Est, cette attitude frileuse s'explique également par les possibles conséquences de cet élargissement de type 'big bang' sur la cohésion interne de l'Union, aussi bien en termes économiques que politiques et culturels. En RFA aussi, les interrogations gagnaient en importance au fur et à mesure que la date fatidique s'approchait. Les coûts budgétaires de ce bouleversement, mais surtout la perspective d'une libre circulation des salariés prenaient de plus en plus de place dans les discours politiques, notamment après l'arrivée au pouvoir de la coalition rouge-verte sous le Chancelier Schröder en 1998. Toutefois, le gouvernement fédéral œuvra en faveur d'une conclusion des négociations d'adhésion tout en espérant que la réforme institutionnelle de l'Union pour garantir son fonctionnement futur se ferait après l'entrée des dix candidats en 2004 - un pari risqué.

Aujourd'hui, les perspectives d'un élargissement futur de l'Union ne sont plus un point de friction entre la France et la RFA (Lefebvre/von Oppeln, 2010). Sur la question de l'adhésion de la Turquie, nous pouvons constater des situations comparables des deux côtés : une opinion publique très largement hostile à l'entrée de la Turquie ; les partis de la droite modérée, la CDU/CSU, I'UMP et les centristes français, clairement opposés, tandis que le SPD et le PS sont majoritairement en faveur de cet élargissement tout en gardant un profil bas sur
Intégration des PECO

Adhésion de la Turquie? 
cette question qui les divise à l'intérieur et qui peut irriter leurs électeurs. Les clivages s'expriment donc à l'intérieur des deux pays et non pas entre leurs gouvernements respectifs. Le gouvernement allemand adopte une position prudente et dilatoire pour faciliter le management des conflits à l'intérieur de la coalition dont le partenaire libéral, le FDP, est plus ouvert à l'élargissement que la CDU/CSU (comme l'était déjà le SPD au moment de la grande coalition de 2005 à 2009). Le président Sarkozy a certes adopté une position plus tranchée contre l'entrée de la Turquie, mais sans pour autant bloquer la poursuite des négociations qui pourraient déboucher sur un partenariat privilégié entre la Turquie et l'Union.

Un autre défi de taille au plan des relations extérieures de l'Union fut certainement la crise irakienne en 2003. La France et l'Allemagne se sont saisies de cette occasion - la crise avait éclaté dans le contexte de la commémoration du $40^{\mathrm{e}}$ anniversaire du Traité de l'Elysée - pour 'revigorer' leur coopération bilatérale et pour monter une opposition diplomatique frontale contre les projets de l'administration Bush de lancer une offensive militaire et de renverser le régime de Saddam Hussein. Cet épisode a montré les limites de l'influence commune sur le partenaire américain, mais surtout les limites d'un leadership franco-allemand au sein de l'Union européenne en matière de politique étrangère dans des cas de figure où les relations des pays membres avec les Etats-Unis entrent en jeu (voir Müller-Brandeck-Bocquet, 2003). La RFA et surtout la France ont dû se rendre à l'évidence que l'Union élargie est plus atlantiste que l'Union des 12 ou des 15. Cette crise a provoqué un clivage d'une ampleur extraordinaire au sein de l'Union, et les présumés 'leaders' de l'Union que sont la France et la RFA ont dû faire l'expérience amère qu'ils n'avaient pas assez de «followers ». La légitimité de leur prétention de leadership européen fut sérieusement remise en question, surtout par les nouveaux pays membres qui ne partagent pas l'idée de la légitimité historique d'un leadership franco-allemand (voir à ce propos Schild, 2010).

\section{Les défis d'avenir de l'Union}

Quel peut être le rôle des deux voisins par rapport aux défis auxquels l'Union se voit confrontée aujourd'hui ? Sans prétendre à l'exhaustivité, on peut citer trois défis majeurs :

- Le développement d'une approche européenne plus coordonnée, voire commune sur les dossiers de la politique énergétique (y compris la sécurité de l'approvisionnement) et de la lutte contre le changement climatique.

- L'évolution de I'Union vers un acteur international à la hauteur des enjeux mondiaux et capable d'apporter sa contribution au maintien et au développement d'éléments vigoureux d'un ordre international.

- Maitriser la crise de l'Euro et garantir la survie durable de la zone euro.

France et RFA : un rôle proactif en matière de réchauffement climatique...
Au cours des dernières années, les deux pays se sont montrés résolus et capables de faire avancer l'agenda européen en matière de lutte contre le réchauffement climatique (à propos de la capacité des deux pays à faire face aux défis d'avenir dans plusieurs domaines, voir Demesmay/Marchetti, 2010). Le rôle proactif qu'ils ont joué tous les deux au cours de leurs dernières présidences de l'Union européenne en 2007 (RFA) et 2008 (France) en témoigne. Bien sûr, le zèle sur ce dossier semble quelque peu freiné depuis 2008 sous l'impact de la crise financière et économique et sous l'effet de l'échec des négociations sur un processus post-Kyoto à Copenhague en décembre 2009. Et les deux pays mettent plus l'accent aujourd'hui sur la préservation de leur tissu industriel qui pourrait s'effriter dans le cas d'un renchérissement unilatéral des coûts de l'énergie au sein de l'Union européenne, mais non pas du côté de ses concurrents économiques. II n'empêche, leur volonté commune et le soutien des sociétés et des forces politiques portent à croire qu'ils continueront à jouer un rôle proactif sur ce dossier. 
Les choses sont différentes quant à une politique énergétique plus coordonnée, voire intégrée. Le France et la RFA ont limité, en 2009, la portée des plans de la Commission pour libéraliser le marché intérieur de l'énergie. Ils ont bloqué une séparation claire entre la production et la distribution d'électricité afin de préserver les intérêts de leur grands producteurs et distributeurs d'énergie sur leurs marchés oligopolistique (RFA) et monopolistique (France) - au détriment du consommateur et au détriment d'un vrai marché commun de l'énergie. Leurs politiques énergétiques extérieures privilégient la voie bilatérale, notamment dans leurs relations énergétiques avec la Russie, le projet le plus important étant le gazoduc Nord Stream dans la mer Baltique auquel coopèrent E.ON et BASF/ Wintershall du côté allemand, et GDF Suez du côté français (depuis 2010). Cette approche ne facilite guère une politique extérieure cohérente de l'Union dans le domaine de ses relations énergétiques avec des pays fournisseurs.

La capacité et la volonté des deux pays à promouvoir un rôle actif et un profil plus visible de l'Union sur la scène internationale ne vont donc pas de soi. Elles sont également limitées par les différences persistantes des cultures politiques et stratégiques en matière de politique étrangère. Ni les élites politiques ni la population allemandes ne sont prêtes à accepter le même fardeau financier pour l'outil militaire, ni les mêmes risques quant à l'emploi de la force armée, que les élites et la population françaises. Les difficultés à préserver un consensus politique au sein du Bundestag sur la poursuite de l'engagement militaire allemand dans le cadre de l'ISAF en Afghanistan en témoignent. Pour développer les capacités militaires et d'intervention sur des théâtres extérieurs, la France se tourne aujourd'hui plutôt vers le Royaume-Uni avec lequel elle vient de conclure un traité bilatéral important en 2010. Celui-ci donne un signal fort aux Allemands et reflète un certain scepticisme français quant aux chances de développer la dimension miliaire et de défense au niveau multilatéral dans le cadre de l'Union européenne (voir Kempin/Mawdsley/Steinicke, 2010).

Le défi européen crucial à l'heure actuelle, et qui risque de dominer l'agenda européen pour un certain temps, est certainement celui de la gestion de la crise de la dette souveraine et de la réforme de la gouvernance économique de la zone euro. Si jamais le projet politique de l'Union monétaire échouait dans les années qui viennent, cet échec serait lourd de conséquences pour l'ensemble de la construction européenne. Un éclatement de la zone euro mettrait en danger le marché unique, saperait la volonté des pays membres à respecter des règles et des disciplines européennes inscrites dans d'autres politiques publiques intégrées, et mettrait les relations bilatérales franco-allemandes à rude épreuve.

En analysant les réactions franco-allemandes face à ce défi majeur, nous pouvons constater un parallèle par rapport à leur réaction face au choc de la crise financière internationale en 2008. Dans les deux cas, l'urgence d'une action européenne fut perçue plus clairement - et beaucoup plus tôt - à Paris. Dans le premier cas, il s'agissait de la coordination européenne des mesures de sauvetage du secteur bancaire et d'une politique vigoureuse de relance économique (voir Schild, 2008 ; Kaufmann/Uterwedde, 2010). Dans le second cas, les tergiversations allemandes avant les élections régionales de la Rhénanie du NordWestphalie en mai 2010, qui ont retardé les décisions européennes sur un plan de sauvetage pour la Grèce, ont produit des irritations profondes à Paris et ailleurs en Europe. Néanmoins, après les réactions divergentes au début de ces deux crises, les zones de convergence franco-allemandes et la coordination bilatérale sont devenues plus visibles par la suite. II y a surtout un consensus sur l'essentiel : l'Union doit mettre en œuvre tous les moyens disponibles pour éviter un éclatement de la zone euro. Malgré une opinion publique et des élites très sceptiques, le gouvernement allemand a accepté un changement implicite des traités européens en donnant son accord à des plans de sauvetage pour la Grèce et pour l'ensemble de la zone euro qui frôlent la violation de la fameuse clause de " no bail out » de l'article 125 du Traité sur le fonctionnement de l'UE. Et il a mis tout le poids politique de la RFA derrière sa proposition de changer
... mais pas de politique énergétique

Divergences de vues en matière de défense

Défi crucial :

soutenabilité de la zone euro 
Quelle gouvernance économique?

Convergence sous la pression des marchés de nouveau les traités afin de permettre la pérennisation d'un mécanisme de sauvetage au-delà de 2013 pour des pays de la zone euro dont l'accès aux marchés financiers serait bloqué faute de confiance des investisseurs privés.

Bien sûr, les différences d'approche de la France et de l'Allemagne, héritées du passé et qui reflètent les expériences et des cultures économiques différentes (voir Pisani-Ferry, 2006) n'ont pas disparu. Elles resurgissent régulièrement quand il s'agit de (re-)définir les éléments d'une gouvernance économique de la zone euro pour mettre celle-ci à l'abri de séismes du type observé en 2010. La RFA poursuit toujours une approche qui met l'accent sur une redéfinition des règles du Pacte de stabilité et de croissance, ainsi que sur des règles nouvelles pour évaluer et garantir le niveau de compétitivité des pays membres de la zone euro. Et l'influence de l'école de pensée ordo-libérale est visible quand le gouvernement allemand veut se servir de la force disciplinatrice des marchés financiers pour mettre sous pression des Etats membres poursuivant des politiques budgétaires et fiscales irresponsables, mais aussi quand elle met l'accent sur la responsabilité des investisseurs privés. Ceux-ci doivent être confrontés au risque de perdre une partie de leurs investissements en cas de défaut souverain. La France, quant à elle, défend toujours la primauté du politique face aux forces des marchés et veut garantir que les décideurs politiques, et non pas la Commission et la BCE, aient le dernier mot dans la gestion des crises et dans l'interprétation des règles pour coordonner les politiques budgétaires. Néanmoins, nous pouvons constater un certain rapprochement. Du côté français, la nécessité de muscler le Pacte de stabilité et son bras préventif est reconnue, et Paris accepte désormais une modification du mode de décision politique au cours de la procédure de déficit excessif pour faciliter des sanctions à l'encontre des 'pécheurs' - sans pour autant souscrire à l'idée d'une sanction quasi-automatique. Du côté allemand, l'idée d'une coordination des politiques budgétaires et fiscales, mais aussi des politiques sociales à l'intérieur de la zone euro semble faire son chemin (Schäuble, 2011).

Tout au long de ce débat sur les réformes des règles qui régissent la zone euro, les deux pays ont su mettre tout leur poids économique dans la balance pour influer sur les choix européens. La manière quelque peu cavalière dont ils ont confronté les partenaires aux résultats de leurs consultations bilatérales à Deauville en octobre 2010 pour les leur imposer ensuite a certainement laissé des traces. Mais les deux pays représentent environ $48 \%$ du PIB de la zone euro (27\% pour la RFA et $21 \%$ pour la France), et leur souscription au capital de la BCE représente également $48 \%$ du total des pays de la zone euro. Ce sont donc les deux qui se portent garants, en cas de crise, pour la moitié des crédits mis à la disposition de pays sans accès aux marchés financiers privés. Leur «power of the purse" leur donne donc un poids particulier dans le processus de redéfinition du dispositif de la gouvernance de la zone euro.

Les opérations de sauvetage pour la zone euro en 2010 et les débats sur la réforme de la gouvernance économique européenne étaient marqués par une certaine asymétrie au sein du « couple » franco-allemand. Les hésitations de la Chancelière allemande au début de la gestion de la crise grecque ont fixé le rythme de la réaction européenne. Plus tard, le gouvernement allemand fut non seulement plus actif que la France en tant que force de proposition, mais il a également su imposer certains éléments de son approche à un partenaire français réticent: l'implication du FMI dans les plans de sauvetage, le caractère strictement intergouvernemental du fonds de stabilisation financière, le refus de tout projet d'émettre des Eurobonds, l'idée de faire partager le risque d'un défaut souverain par les investisseurs privés, et finalement le nouveau changement des traités européens. En plus, l'Allemagne a pris en 2010 la décision non concertée - d'un programme d'austérité budgétaire. Cette approche allemande a immédiatement augmenté la pression sur ses partenaires, dont la France, de suivre son exemple pour éviter que les primes de risque exigées par les marchés pour leurs obligations d'État n'augmentent sensiblement. 
La politique allemande au cours de la tempête que la zone euro est en train de traverser a fait naître un certain malaise à Paris (voir par exemple Vedrine, 2010 ; Gougeon, 2010). La cause profonde de ce malaise réside dans la perception française d'un risque de découplage économique entre les deux pays qui coïncide avec une diplomatie européenne d'une RFA qui défend ses intérêts sans complexes.

\section{Le défi bilatéral : un décrochage franco-allemand?}

Certes, le prix économique et budgétaire que la RFA a dû payer pour l'intégration économique, sociale et politique de l'ex-RDA fut largement sous-estimé en France (tout comme en RFA), et les ressources de puissance à la disposition de l'Allemagne clairement surestimées. Le fardeau énorme des transferts financiers vers les nouveaux Länder a sapé pendant une décennie la compétitivité allemande, et la part de marché de l'Allemagne sur le marché mondial a baissé pendant quelques années (voir REA 39/1998). Depuis 2000, la donne a changé. «A partir du début des années 2000, une divergence de compétitivité sans précédent historique apparaît au détriment de la France et depuis, l'écart de compétitivité s'accroît ", tel est le constat alarmant d'une étude récente commandée par le ministère de l'Economie français (COE-Rexecode, 2011). Les écarts entre la France et la RFA en termes de compétitivité se creusent.

Si on indexe l'évolution des coûts unitaires du travail sur l'année 2000 (2000 = $100)$, on constate clairement ce décrochage entre la RFA et les partenaires européens, dont la France, sur cet indicateur clé de la compétitivité internationale. En ce qui concerne la balance des comptes courants, la même tendance est facilement perceptible.
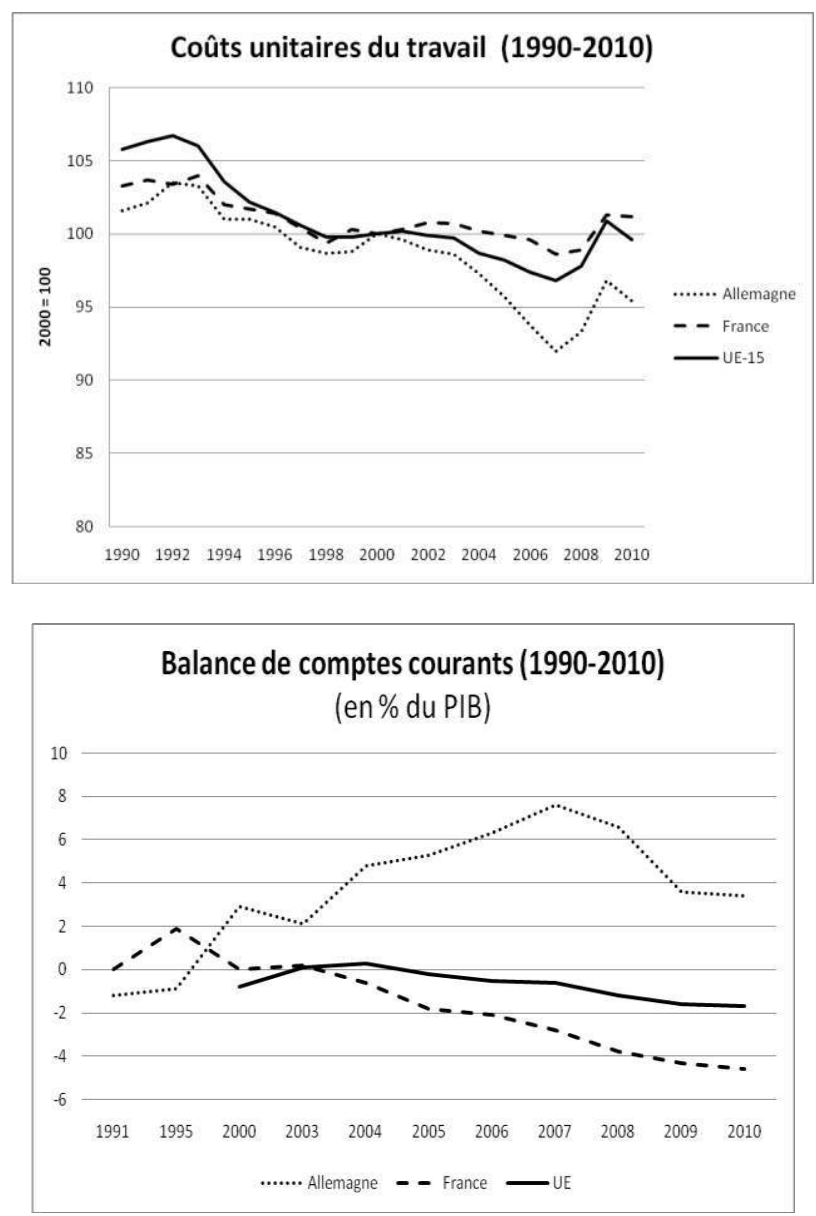

Source : Eurostat. NB : Je remercie Thomas Oberkirch pour la recherche de ces données et leur mise en graphique. 
Un décrochage avant tout structurel...
... et qui présente des risques pour la stabilité de l'Union
En ce qui concerne les taux de croissance, par contre, un écart franco-allemand est un phénomène très récent. Mais il pourrait persister au cas où la demande des pays émergents pour des biens d'équipement allemands resterait soutenue et doperait les exportations et la croissance allemandes dans les années qui viennent. Le décrochage franco-allemand a en effet pour principale raison « les disparités structurelles des deux économies » (Lasserre, 2007).

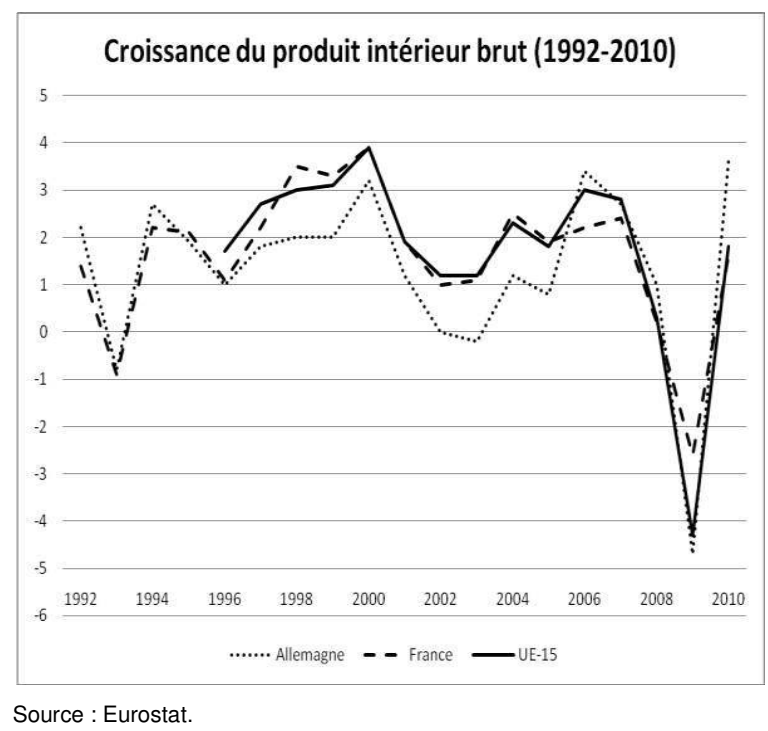

A considérer ce décalage entre les deux pays en matière de taux de croissance, de niveau de chômage, de coûts unitaires du travail, de la balance des paiements et de leurs situations budgétaires, on peut se demander si nous ne vivons pas une sorte de retour vers une situation semblable à celle des années 1980. Dans la perception française de l'époque, c'était la politique monétaire allemande, donc la Bundesbank, qui faisait le bon et le mauvais temps au sein du système monétaire européen (SME). L'Allemagne exerçait donc une forte pression d'adaptation sur les politiques monétaires et fiscales des pays partenaires à travers les mécanismes du SME. Vingt ans plus tard, c'est de nouveau le modèle de stabilité allemand qui donne le «la » au sein de la zone euro (Kotz, 2009). Les politiques budgétaires et fiscales des 16 partenaires qui partagent avec elle l'euro en 2011 sont jugées par les marchés financiers à l'aune de l'écart de leurs déficits, de leurs dettes, de leur compétitivité et de leur croissance par rapport aux performances allemandes. Ces écarts déterminent le niveau de leurs primes de risque sur les marchés des obligations souveraines, qui se compare toujours au niveau allemand. Une telle évolution et de telles perceptions comportent évidemment de lourds risques aussi bien pour la politique européenne de la RFA que pour la solidité de la relation privilégiée francoallemande et pour la cohésion de la zone euro.

Après avoir abandonné sa souveraineté en matière monétaire, la France se voit confrontée aujourd'hui à la réalité d'une marge de manœuvre extrêmement limitée pour sa politique budgétaire et fiscale, donc du deuxième pilier de toute politique macroéconomique. D'où la priorité et l'accent qu'elle met sur une coordination renforcée des politiques économiques, surtout fiscales, des pays de la zone euro. II est politiquement aussi difficile pour la RFA en 2011 de se soustraire à cette demande française qu'il l'était en 1988 de refuser tout progrès vers I'UEM.

La Chancelière Merkel a réagi face à cette situation, d'abord en adoptant le terme de "gouvernement économique »-sans pour autant souscrire à toutes les idées que ce concept (assez flou) peut contenir -, puis en lançant l'idée, soutenue par la France, d'un pacte européen pour la compétitivité. Celui-ci aurait pour objectif de coordonner des politiques au-delà des seules politiques budgé- 
taires, notamment en matière de taxation, de politique sociale, mais aussi salariale. II convient à l'idée française d'une gouvernance de la zone euro au niveau de ses 17 pays membres et pourrait déboucher sur une coopération renforcée d'un type strictement intergouvernemental, c'est-à-dire en écartant la Commission européenne. Toute la question est de savoir si les partenaires suivront cette voie indiquée par la France et la RFA et si les formes de coordination prévues sont vraiment à même de resserrer les écarts de compétitivité qui se sont fait jour depuis le lancement de l'euro. Dans ces domaines qui touchent à ses ressources financières et sa compétitivité - mais non pas dans d'autres ! -, la RFA est effectivement devenue la "Zentralmacht », donc incontournable pour toute recherche de compromis européens.

UN CERTAIN NOMBRE D'INQUIÉTUDES EXPRIMÉES AU MOMENT DE L'UNIFICATION allemande et que nous avons évoquées plus haut se sont révélées sans fondement. L'ampleur du défi de l'absorption économique de l'ex-RDA a certainement été sous-estimée. Le gain suite à l'unification, en termes de ressources de pouvoirs de cette Allemagne unifiée, est resté très relatif. La population allemande représente aujourd'hui à peine plus de $1 \%$ de la population mondiale $(1,21 \%$ en 2009 par rapport à $1,50 \%$ en 1990) et a tendance à baisser fortement.

La RFA n'a nullement abandonné son ancrage solide au sein de I'Union européenne au profit d'une politique nationale en quête d'une zone d'influence en “Mitteleuropa ". S'il y a bien eu des exemples de décisions solitaires en matière de politique étrangère et européenne, ils sont restés l'exception à la règle, ainsi la reconnaissance précoce de la Slovénie et de la Croatie en 1991, le refus unilatéral de tout engagement militaire en Irak en 2002/03 ou encore l'interdiction unilatérale des ventes d'actions à découvert en 2010. La diplomatie allemande est donc restée fermement ancrée dans des cadres multilatéraux. Quand des défis nouveaux se présentent au niveau international - la lutte contre le terrorisme dans l'après « 9 septembre », la stabilisation d'Etats fragiles à travers le monde, la lutte contre la prolifération nucléaire ou encore contre le réchauffement climatique - la RFA a toujours gardé ses réflexes de coordination au niveau européen. Et la relation privilégiée avec la France reste toujours la référence centrale de sa politique européenne.

Ce qui a changé, par contre, c'est la manière dont la RFA défend ses intérêts au sein de l'Union européenne, mais aussi vis-à-vis de la France. La politique extérieure allemande a toujours cherché sa voie entre les pôles d'une politique de "Selbstbeschränkung " (autolimitation) et de "Selbstbehauptung » (affirmation de soi), la première étant la base de la seconde (Haftendorn, 2001). Le curseur entre ces deux pôles s'est déplacé quelque peu vers le second. La puissance "apprivoisée» a adopté plus souvent des types de comportement d'une puissance "normalisée " (voir l'excellente analyse sur le changement du rôle de l'Allemagne au sein de l'Union européenne de Bulmer/Paterson, 2010). Ceci est surtout vrai dans les domaines où la RFA peut s'appuyer sur son poids économique et ses ressources financières à l'intérieur de I'Union, dans la politique budgétaire européenne, dans le domaine monétaire et la gestion de la crise de l'euro. Ce comportement rend la gestion des relations bilatérales avec la RFA plus complexe et plus difficile pour la France. Mais les tentatives de créer un contrepoids contre l'influence de la RFA au sein de l'Union, tel le projet de Nicolas Sarkozy de créer une Union de la Méditerranée qui exclut la RFA, n'ont mené nulle part. Les deux pays restent l'un pour l'autre le partenaire incontournable dont ils ont besoin dans la poursuite de leurs intérêts les plus importants au niveau européen.

Une crainte évoquée plus haut est celle d'une dilution du poids réuni de la France et de la RFA au sein de l'Union européenne élargie. Cette thèse doit, elle aussi, être nuancée. Certes, les prétentions franco-allemandes en matière de leadership européen se sont parfois heurtées à une fin de non-recevoir, surtout dans l'épisode de la guerre irakienne en 2003. Et leur poids dans les décisions 
législatives quotidiennes à Bruxelles n'est plus le même que par le passé, suite à l'élargissement et à la montée en puissance du Parlement européen. II n'empêche, quand il s'agit de prendre des décisions de première importance - modification des traités, budget pluriannuel, gestion de crise de l'euro mobilisant d'importantes ressources financières -, les deux partenaires se retrouvent toujours au centre des décisions européennes. La concomitance de la croissance du nombre d'Etats membres et la perte d'influence de la Commission ont pour conséquence de donner un caractère plus informel et plus intergouvernemental aux négociations et à la préparation des décisions européennes. Ces évolutions créent des opportunités pour les grands pays qui se retrouvent aux nœuds de communication, et donc au centre des décisions européennes.

Quel serait l'alternative à un rôle particulier de la France et de la RFA qui assument leur responsabilité particulière pour garantir l'acquis des politiques européennes et pour la construction européenne dans son ensemble ? Un leadership allemand en Europe est certainement hors de portée pour la RFA et inacceptable pour ses partenaires, en premier lieu pour la France qui a déjà du mal à se faire à l'idée d'un leadership bilatéral d'un type asymétrique. L'alternative, c'est donc une Europe sans leadership aucun - une perspective plutôt alarmante, vu l'ampleur des défis auxquels l'Union se voit aujourd'hui confrontée.

\section{Indications bibliographiques}

-Bıtsch M.-T. (dir.), Le couple France-Allemagne et les institutions européennes. Une postérité pour le plan Schuman, Bruxelles, 2001

-Bozo F., Mitterrand, la fin de la Guerre froide et l'unification allemande. De Yalta à Maastricht, Paris, 2005

- Bulmer S., Paterson W., "Germany and the European Union : from 'tamed power' to normalized power? ", in International Affairs, vol. 86, $n^{\circ} 5,2010$

- Coe-ReXeCode, Mettre un terme à la divergence de compétitivité entre la France et l'Allemagne, janvier 2011 (www.coe-rexecode.fr)

-Defrance C., Pfeil U. (dir.), Der Elysée-Vertrag und die deutsch-französischen Beziehungen 1945 - 1963 - 2003, Munich, 2005

- Demesmar C., Marchetti A. (dir.), La France et l'Allemagne face aux crises européennes, Bordeaux, 2010

-Deubner C., «Frankreich in der Osterweiterung der EU, 1989-1997 », in Politische Studien, $\mathrm{n}^{\circ} 363,1999$

-Dyson K. F., Featherstone K., The road to Maastricht. Negotiating economic and monetary union, Oxford, 2010

- HAFTENDORN H., Deutsche Außenpolitik zwischen Selbstbeschränkung und Selbstbehauptung. 1945 - 2000, Stuttgart, 2001

-Gougeon J.-P., " Non-dit franco-allemand. Le déséquilibre ne cesse de croître entre les deux pays », Le Monde, 06-03-2010

-KAUDER V., «Europa muss sich konsolidieren », Frankfurter Allgemeine Zeitung, 09-05-2009

- KaUfmann P., UterWedde H., "La France et l'Allemagne face à la crise de l'euro. A la recherche de la convergence perdue ", Visions franco-allemandes, n¹7, IFRI, juillet 2010 (www.ifri.org/ downloads/vfa17kaufmannuterwedde.pdf)

-Kempin R., MAWDSLey J., Setinicke S., " Abkehr von der GSVP ? Französisch-britischer Bilateralismus in der Sicherheits- und Verteidigungspolitik ", SWP Aktuell, $n^{\circ} 81$, Stiftung Wissenschaft und Politik, Berlin, novembre 2010

-Kotz H.-H., « 60 ans de Loi fondamentale, 61 ans de Deutsche Mark », Regards sur l'économie allemande, $\mathrm{n}^{\circ}$ 91, mai 2009

-LASSERRE R., "Surmonter le décrochage franco-allemand ", Regards sur l'économie allemande, $\mathrm{n}^{\circ} 81$, mai 2007

- LASSERRE R., "Un viatique pour Lisbonne », Regards sur l'économie allemande, n॰92, juillet 2009

-MAgnette P., Kalypsos N., « Grands et petits Etats dans l'Union européenne. Réinventer l'équilibre ", Paris, Notre Europe, Etudes et Recherches n²5, mai 2003

- MAZucelli C., France and Germany at Maastricht. Politics and negotiations to create the European Union, New York, 1997

-MARSH D., The euro. The politics of the new global currency, New Haven, Connecticut, 2009

-MARTENS S. (dir.), L'Allemagne et la France. Une entente unique pour l'Europe, Paris, 2004 


\section{EUROPE}

-Maull H.W., StaHL B., « Krisenmanagement im Jugoslawienkonflikt. Deutschland und Frankreich im Vergleich ", in MeIMETH M. et SCHILD J. (dir.), Die Zukunft von Nationalstaaten in der europäischen Integration. Deutsche und französische Perspektiven, Opladen, 2002

- Meimeth M., SCHILD J. (dir.), Die Zukunft des Nationalstaats in der europäischen Integration. Deutsche und französische Perspektiven, Opladen, 2002

-MÜLleR-BRANDECK-Bocquet G., « Der deutsch-französische Gleichklang in der Irak-Krise - Ausgangspunkt für weitere außen- und sicherheitspolitische Gemeinsamkeiten? ", in Politische Studien, $n^{\circ} 392$, novembre-décembre 2003

-MüLleR-Graff P.-C., "L'arrêt de Karslruhe sur le Traité de Lisbonne ", in Regards sur l'économie allemande, $n^{\circ}$ 92, juillet 2009

- PIsani-Ferri J., "Only One Bed for Two Dreams : A Critical Retrospective on the Debate over the Economic Governance of the Euro Area ", Journal of Common Market Studies, Vol. 44, $n^{\circ} 4$, 2006

-SchäUBlE W., «Für eine bessere Verfassung Europas », Frankfurter Allgemeine Zeitung, 27-012011.

-ScHILD J., «,Den Rhein vertiefen und erweitern'. Deutsch-französische Beziehungen nach dem Nizza-Gipfel ", in Aktuelle Frankreich-Analysen, n 17, février 2001

- ScHILD J., «La France, l'Allemagne et la Constitution européenne : un bilan mitigé, un leadership contesté ", in Notes du Cerfa, Paris, mars 2004 (www.ifri.org/files/Cerfa/NoteCerfa_10.pdf)

- ScHILD J., « "La force de l'imprévisible : la présidence française de l'UE face à la crise financière. Un point de vue allemand ", in Regards sur l'économie allemande, n 89, décembre 2008

- ScHILD J., "Mission impossible. The Potential for Franco-German Leadership in the Enlarged EU ", in Journal of Common Market Studies, Vol. 48, n 5, 2010

-Scholl B., Europas symbolische Verfassung. Nationale Verfassungstraditionen und die Konstitutionalisierung der EU, Wiesbaden, 2006

-SchwaRz H.-P., Die Zentralmacht Europas. Deutschlands Rückkehr auf die Weltbühne, Berlin, 1994

-StaRK H., "La France et l'Allemagne face a l'Est : le cas yougoslave », in CIRAC et al. (dir.), Agir pour l'Europe. Les relations franco-allemandes dans l'après-guerre froide, Paris, 1995

-StARK H., "Présidence allemande de l'UE : de la 'racine carrée' à la quadrature du cercle ", in Regards sur l'économie allemande, $\mathrm{n}^{\circ} 82$, juillet 2007

-VÉDRINE H., «France-Allemagne, le malaise », Le Monde, 29-06-2010

-Webber D. (dir.), The Franco-German relationship in the European Union, Londres, 1999

-ZIEBURA G., Die deutsch-französischen Beziehungen seit 1945. Mythen und Realitäten, Stuttgart, 1997. 
S. HAZOUARD, R. LASSERRE,

H. UTERWEDDE (dir.),

Les politiques d'innovation coopérative

en Allemagne et en France.

Expériences et approches comparées.

Cergy-Pontoise, décembre 2010, $260 \mathrm{p}$.

ISBN 978-2-905518-39-2, $25 €$.

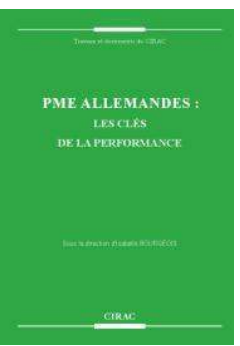

du CIRAC

I. BOURGEOIS, R. LASSERRE, La République fédérale d'Allemagne.

Chronique politique, économique et sociale 1949-2009.

Cergy-Pontoise, août 2009, 128 p.,

ISBN 978-2-905518-37-8, $25 €$.

(chronique illustrée, quadrichromie)
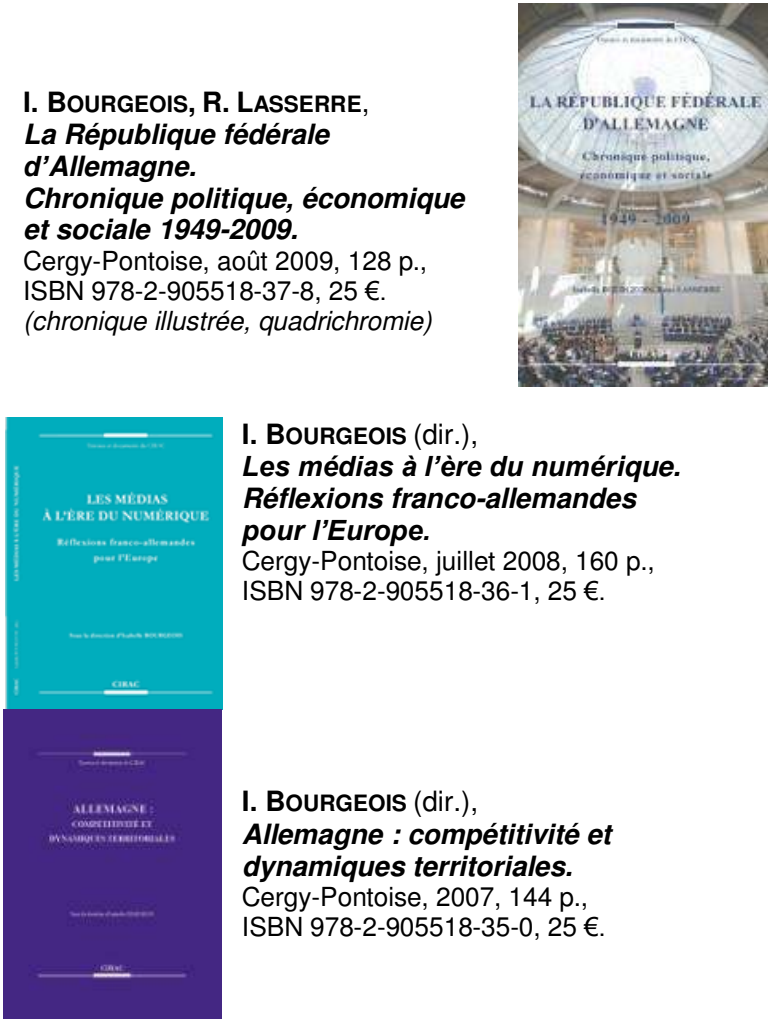

I. BOURGEOIS (dir.),

Les médias à l'ère du numérique.

Réflexions franco-allemandes

pour l'Europe.

Cergy-Pontoise, juillet 2008, $160 \mathrm{p}$

ISBN 978-2-905518-36-1, $25 €$.

I. BOURGEOIS (dir.),

Allemagne : compétitivité et

dynamiques territoriales.

Cergy-Pontoise, 2007, $144 \mathrm{p}$

ISBN 978-2-905518-35-0, $25 €$

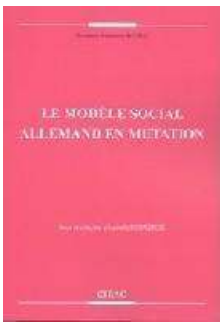

I. BOURGEOIS (dir.),

Le modèle social allemand en mutation

Cergy-Pontoise, 2005, 208 p.,

ISBN 2-905518-33-2, $25 €$.

I. BOURGEOIS, (dir.),

Allemagne 2001

Regards sur une économie en mutation.

Levallois-Perret, 2000, $416 \mathrm{p}$.,

ISBN 518-30-8, $32 €$

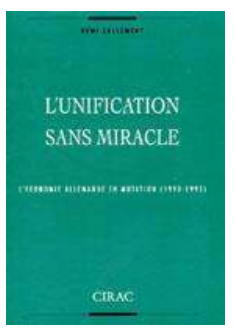

R. LALLEMENT,

L'unification sans miracle.

L'économie allemande

en mutation (1990-1995).

Levallois-Perret, 1995, $176 \mathrm{p}$.

ISBN 2-905518-28-6, 18,29€

P. Commun (dir.),

L'ordolibéralisme allemand. Aux sources de l'économie sociale de marché. Cergy-Pontoise, 2003, $274 \mathrm{p}$., ISBN 2-905518-31-6, 32 €.28-6, 18,29€.
I. BOURGEOIS (dir.), PME allemandes: Les clés de la performance rgy-Pontoise, août 2010, $144 \mathrm{p}$. ISBN 978-2-905518-38-5, $20 €$.

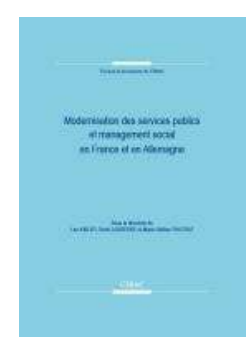

L. KIBLER, R. LASSERRE, M.-H. PAUTRAT (dir.) Modernisation des services

France et en Allemagne.

Cergy-Pontoise, 2007, $144 \mathrm{p}$. ISBN 978-2-905518-34-0, $20 €$.
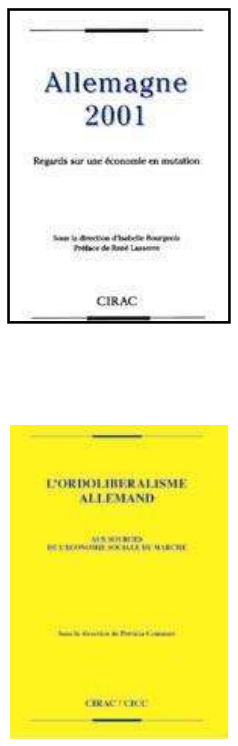

Liste complète des publications sur : www.cirac.u-cergy.fr 